\title{
QUADRATIC EXTENSIONS OF LINEARLY COMPACT FIELDS
}

\author{
BY \\ RON BROWN(') AND HOYT D. WARNER( $\left.{ }^{2}\right)$
}

\begin{abstract}
A group valuation is constructed on the norm factor group of a quadratic extension of a linearly compact field, and the norm factor group is explicitly computed as a valued group. Generalizations and applications of this structure theory are made to cyclic extensions of prime degree, to square (and $p$ th power) factor groups, to generalized quaternion algebras, and to quadratic extensions of arbitrary fields.
\end{abstract}

Introduction. Let $F$ be a valued field, with valuation $v$ and valuation ring $R_{v}$, such that the additive group of $F$ is topologically complete for every (not necessarily Hausdorff) group topology admitting a family of ideals of $R_{v}$ as a neighborhood base at 0 . This condition on a valued field is equivalent to Lefschetz's linear compactness (as an $R_{v}$-module) [13], Krull's maximality [12], and Fleischer's ultracompleteness [6]. These concepts were introduced to generalize the classical local fields. For facts about linearly compact fields (and further equivalent definitions) see [2], [11], [15], [18], [19].

Assuming that $F$ does not have characteristic two and that the residue class field $k_{v}$ of $v$ is perfect if it has characteristic two, we show the field valuation $v$ naturally induces a valuation on the norm factor group of any quadratic extension of $F$ (see $\S 1$ for valuations on groups). We then compute the structure of these factor groups as valued groups. We generalize and apply this computation to the square factor group of $F$, to generalized quaternion division algebras over $F$ (and in particular their norm factor groups), to quadratic extensions of not necessarily linearly compact fields, and to the norm factor groups of arbitrary cyclic extensions of prime degree.

Presented to the Society, January 22, 1971; received by the editors June 1, 1970.

AMS 1969 subject classifications. Primary 1398, 1067, 1270; Secondary 1069, 1570, 1245, 1646.

Key words and phrases. Linearly compact field, maximal field, valuation, quadratic field extension, norm factor group, square factor group, ramification, generalized quaternion algebra, quadratic forms, valued group, filtered group, ultracomplete valued group, graded group, well ordered product, Hahn product, Henselian field.

( ${ }^{1}$ Supported by an NSF graduate fellowship, an ONR, NRC associateship, and a postdoctoral fellowship at Simon Fraser University (partially funded by the Canadian National Research Council).

${ }^{(2)}$ Supported by an NSF graduate fellowship, and a post-doctoral fellowship at the University of Illinois. 
As abstract groups the above square and norm factor groups are simply abelian groups of prime exponent (i.e. vector spaces over a finite field) and hence are determined by their cardinalities. The valuations put on these groups considerably enrich their structure and apply directly (see $\$ 4$ ) to the study of generalized quaternion algebras over $F$ (clarifying the situation even for the classical local fields where all the factor groups are finite). This enrichment is of main importance when the residue class field of $v$ has characteristic two and the extension of $F$ is ramified-the "wildly ramified" case; in tamely ramified extensions the valuations are transparent and the cardinality of the factor groups well understood (cf. [16]). See $\$ 1$ for further comments on this subject.

We now outline the paper. We denote the multiplicative and additive groups of a field $E$ by $E^{\times}$and $E^{+}$respectively, and we write the value group $\Gamma_{v}=v\left(F^{\times}\right)$of $v$ additively.

In $\S 1$ we state the main results of the paper. We describe explicitly how the structure as a valued group of the square factor group $F^{\times} / F^{\times 2}$ of $F$ is determined by $\Gamma_{v}$, the element $v(2)$ of $\Gamma_{v}$, and the residue class field $k_{v}$ of $v$. Next, for any nonsquare $d$ in $F$ we show how the norm factor group $F^{\times} / N K^{\times}$of the extension $K=F[\sqrt{ } d]$ of $F$ is determined (as a valued group) by $\Gamma_{v}, v(2), k_{v}$ and three invariants depending on $d \cdot F^{\times 2}$ (as an element of $F^{\times} / F^{\times 2}$ ): roughly, the image of $d \cdot F^{\times 2}$ in $k_{v}^{\times} / k_{v}^{\times 2}$, the image of $d \cdot F^{\times 2}$ in $\Gamma_{v} / 2 \Gamma_{v}$, and the "quadratic defect" (cf. [16, p. 160]) of $d \cdot F^{\times 2}$.

In $\$ 2$ we discuss filters on groups, especially those induced by valuations and those "ultracomplete" in a natural sense. We apply this discussion in $\S 3$ to prove the theorems stated in the first section; the calculations of this section owe a great debt to the papers of MacKenzie-Whaples and Geissinger [14], [7].

The above structure theory is applied in $\$ 4$ to compute the norm factor group of a generalized quaternion division algebra (i.e. a central division algebra of dimension 4) over $F$, again naturally considered as a valued group. We also observe that certain strong hypotheses on $k_{v}$ and $\Gamma_{v}$ (valid for the nonarchimedean local fields and also used in [7], for example in a calculation of norm factor groups) imply there is a unique quaternion division algebra over $F$. As observed by Kaplansky, this implies that a quadratic form over such $F$ is determined up to equivalence by its degree, discriminant, and Hasse algebra, and any form of degree greater than four is isotropic.

In $\S 5$ we discuss our use of the hypothesis of linear compactness; this gives insight into the ramification theory of quadratic extensions for not necessarily linearly compact $F$ (also see [3], [4]). It is a consequence of our remarks here that in some cases our hypothesis of linear compactness can be weakened to relative completeness (i.e. satisfying Hensel's lemma), e.g. when $\Gamma_{v}$ is an infinite cyclic group or when our quadratic extension is tamely ramified and not immediate.

Finally in $\S 6$ we describe the norm factor group of a cyclic extension of $F$ of prime degree $p$ and the $p$-factor group $F^{\times} / F^{\times p}$ (under hypotheses on $F$ generalizing the case $p=2$ ). 
Our hope is that these calculations will cast some light on the possibilities for the local part of a theory of quadratic forms generalizing the classical theory for global fields [16]. In this direction we notice [4] where, by a suitable completion process, certain linearly compact fields are associated with the finite and infinite primes [9, p. 3] of a field (in such a way as to generalize the completions of global fields at prime spots). As examples of the important role that square and norm factor groups play in the theory of quadratic forms we mention the following:

(1) Theorem 7 of Witt [20] which shows how the Witt group of a field is determined by the norm and square factor groups;

(2) O'Meara [16] where it is remarked that the Hasse-Minkowski theorem follows from the Hasse norm theorem (plus the global squares theorem);

(3) the question of what values a binary quadratic form can assume is quickly reduced (by eliminating trivial cases and completing the square) to computing the set of norms from a particular quadratic extension.

The initial research for this paper took place in the stimulating atmosphere of a seminar conducted by Professor D. K. Harrison at the University of Oregon in 1967-1968; the authors wish to express their warm thanks to him for encouraging their study of these questions.

1. Main results. Throughout this paper, except for $\S \S 5$ and 6 , we assume as in the introduction that $F$ with valuation $v$ is linearly compact, does not have characteristic two, and that the residue class field of $v$ is perfect if it has characteristic two.

We now define the notion of a valuation on an abelian group and give some key examples which motivate and prepare for our main theorems (for these definitions cf. Conrad [5], Gravett [8], Fleischer [6]). Let $\Lambda$ be a linearly ordered set and $G$ an abelian group, written additively. A map $v: G \rightarrow \Lambda \cup\{\infty\}$ is a valuation (or a $\Lambda$-valuation) if and only if for all $a$ and $b$ in $G$ we have $v(a)=v(-a), v(a+b)$ $\geqq \min (v(a), v(b))$, and $v(a)=\infty$ if and only if $a=0$. (Here $\infty$ is a formal symbol with $\infty>\alpha$ for all $\alpha \in \Lambda$.) The following examples are fundamental.

EXAMPLE (A). $v$ is a valuation on the additive group $F^{+}$of $F$.

EXAMPLE (B). $v$ induces a valuation $v^{\times}$on the multiplicative group $F^{\times}$of $F$. Linearly order

$$
\Lambda_{v}=\left\{0^{*}\right\} \cup\left\{\alpha \in \Gamma_{v} \mid \alpha \geqq 0\right\}
$$

(where $0^{*}$ is a formal symbol) by letting $\Lambda_{v} \cap \Gamma_{v}$ inherit its order from $\Gamma_{v}$ and setting $0^{*} \leqq \alpha$ for all $\alpha \in \Lambda_{v}$. Then the map

$$
v^{\times}: F^{\times} \rightarrow \Lambda_{v} \cup\{\infty\}
$$

with $v^{\times}(a)=0^{*}$ if $v(a) \neq 0$ and $v^{\times}(a)=v(1-a)$ if $v(a)=0$ is a valuation.

The valuations we will put on the square and norm factor groups of $F$ will be naturally and directly induced by $v^{\times}$.

EXAMPLE (C). Let $\Delta=\left(D_{\lambda}\right)_{\lambda \in \Lambda}$ be a family of abelian groups indexed over a linearly ordered set $\Lambda$ (i.e. a $\Lambda$-graded group). By the well-ordered product of $\Delta$, 
denoted by $\mathbf{H} \Delta$ or $\mathbf{H}_{\lambda \in \Lambda} D_{\lambda}$, we mean the subgroup of the direct product $\prod D_{\lambda}$ $\left(\lambda \in \Lambda\right.$ ) consisting of those maps $\varphi$ from $\Lambda$ to $\bigcup D_{\lambda}$ with $\varphi(\lambda)=0$ (i.e. the identity of $D_{\lambda}$ ) except on a well-ordered subset of $\Lambda$. The map which sends each $\varphi \in \mathbf{H} \Delta$ to the least $\lambda \in \Lambda$ with $\varphi(\lambda)$ nonzero (i.e., $\varphi(\lambda)$ not the identity of $D_{\lambda}$ ), with $\infty$ the minimum of the empty set, is a valuation on $\mathbf{H} \Delta$ called the canonical valuation.

We can now state the calculation of the square and norm factor groups of $F$. In each case we will explicitly describe a graded group; each theorem will assert that the factor group of $F^{\times}$in question, with a valuation naturally induced by $v^{\times}$ (cf. Example (B) above), is isomorphic as a valued group to the well-ordered product of the appropriate graded group with its canonical valuation (cf. Example (C)).

With $\Lambda_{v}$ defined as in Example (B) above, let $\Delta^{\mathrm{sq}}(F)$ be the $\Lambda_{v}$-graded group given by

$$
\begin{aligned}
\Delta^{\mathrm{sq}}(F)_{\gamma} & =\Gamma_{v} / 2 \Gamma_{v} & & \text { if } \gamma=0 *, \\
& =k_{v}^{\times} / k_{v}^{\times 2} & & \text { if } \gamma=0, \\
& =k_{v}^{+} & & \text {if } 0<\gamma<v(4), \gamma \notin 2 \Gamma_{v}, \\
& =k_{v}^{+} /\left\{a^{2}-a \mid a \in k_{v}\right\} & & \text { if } \gamma=v(4)>0, \\
& =\text { the trivial group } & & \text { otherwise. }
\end{aligned}
$$

Note the third and fourth cases above can arise only if $k_{v}$ has characteristic two.

(1.1) THEOREM. There exists a value-preserving isomorphism

$$
F^{\times} / F^{\times 2} \rightarrow \mathbf{H} \Delta^{\mathrm{sq}}(F)
$$

where the valuation on $F^{\times} / F^{\times 2}$ is the map $v^{\mathrm{sq}}$ into $\Lambda_{v} \cup\{\infty\}$ given by

$$
v^{\mathrm{sq}}\left(d \cdot F^{\times 2}\right)=\max \left\{v^{\times}(a) \mid a \in d \cdot F^{\times 2}\right\}
$$

(and the valuation on the well-ordered product is the canonical one).

This theorem is proved in $\S 3$. Note that it implies that the image of the nonidentity elements of $F^{\times} / F^{\times 2}$ under $v^{\text {sq }}$ is the set of $\lambda \in \Lambda_{v}$ with $\Delta^{\mathrm{sq}}(F)_{\lambda}$ nontrivial, so that when $k_{v}$ does not have characteristic two, 0 and $0^{*}$ are the only possibilities.

Now let $K$ be any quadratic extension of $F$. Let $N: K \rightarrow F$ be the norm map. Let $w$ be the extension of $v$ to $K$ (for the existence and uniqueness of $w$ see Chapter 2 of [19]). We will also denote by $N: k_{w} \rightarrow k_{v}$ the map that $N: K \rightarrow F$ induces on the residue class fields.

(1.2) Proposition And Definition. The set $\left\{w^{\times}(a) \mid a \in K, N a=1\right\}$ has a minimum which we denote by $\lambda=\lambda_{K / F}$.

(It is easy to verify that an element $a \in K^{\times}$is a "distortion constant" in the sense of MacKenzie-Whaples [14] if and only if $w\left(a^{\sigma} a^{-1}-1\right)=\lambda$ where $a^{\sigma}$ is the conjugate of $a$.) 
We define a $\Lambda_{v}$-graded group $\Delta^{N}(K / F)$ by

$$
\begin{aligned}
\Delta^{N}(K / F)_{\gamma} & =\Gamma_{v} / 2 \Gamma_{w} & & \text { if } \gamma=0^{*}, \\
& =k_{v}^{\times} / N k_{w}^{\times} & & \text {if } \gamma=0, \\
& =k_{v}^{+} & & \text {if } 0<\gamma<2 \lambda, \gamma \notin 2 \Gamma_{w}, \\
& =k_{v}^{+} /\left\{a^{2}-a \mid a \in k_{v}\right\} & & \text { if } \gamma=2 \lambda>0, \\
& =\text { the trivial group } & & \text { otherwise. }
\end{aligned}
$$

Again note that the third and fourth cases can occur only if $k_{v}$ has characteristic two; actually we shall see they occur only if $K / F$ is wildly ramified (i.e. $k_{v}$ has characteristic two and $\left(\Gamma_{w}: \Gamma_{v}\right)=2$; see Lemma (3.1) below).

(1.3) THEOREM. There exists a value-preserving isomorphism

$$
F^{\times} / N K^{\times} \rightarrow \mathbf{H} \Delta^{N}(K / F)
$$

where the valuation $v^{N}$ on $F^{\times} / N K^{\times}$is given by

$$
v^{N}\left(b \cdot N K^{\times}\right)=\max \left\{v^{\times}(a) \mid a \in b \cdot N K^{\times}\right\}
$$

for any $b \in F^{\times}$(and $\mathbf{H} \Delta^{N}(K / F)$ has its canonical valuation).

This theorem is proved in $\S 3$. Again notice that the image of $F^{\times} / N K^{\times}$under $v^{N}$ is $\infty$ together with the $\gamma \in \Lambda_{v}$ with $\Delta^{N}(K / F)_{\gamma}$ nontrivial, so that when $K / F$ is not wildly ramified, $\infty, 0^{*}$ and 0 are the only possibilities.

(1.4) Remark. There is a unique coset $d \cdot F^{\times 2}$ in $F^{\times} / F^{\times 2}$ with $K=F[\sqrt{ } d]$. With notation as above we have

$$
v(4)=2 \lambda_{K / F}+v^{\mathrm{sq}}\left(d \cdot F^{\times 2}\right)
$$

(where $0^{*}+a=a \pm 0^{*}=a$ for all $a \in \Lambda_{v} \cup\{\infty\}$ ). It is not hard to verify that when $v(d)=0,\left\{a \in F \mid v(a) \geqq v^{\mathrm{sq}}\left(d \cdot F^{\times 2}\right)\right\}$ is the quadratic defect of $d$ in the sense of O’Meara [16, p. 160].

Let $d \cdot k_{v}^{\times 2}$ denote $\left\{a+\mathfrak{p}_{v} \mid a \in d \cdot F^{\times 2}, v(a)=0\right\}$ (here $\mathfrak{p}_{v}$ denotes the maximal ideal of the valuation ring $R_{v}$ ). Notice $d \cdot k_{v}^{\times 2}$ is either empty or an element of $k_{v}^{\times} / k_{v}^{\times 2}$. We have (here $Z$ denotes the ordinary integers)

$$
\begin{gathered}
2 \Gamma_{w}=2 \Gamma_{v}+Z \cdot v(d)+Z \cdot v^{\mathrm{sq}}\left(d \cdot F^{\times 2}\right), \\
N k_{w}^{\times}=\left\{a \in k_{v}^{\times} \mid \exists b \in k_{v}^{\times 2}, b-a \in d \cdot k_{v}^{\times 2} \cup\{0\}\right\} .
\end{gathered}
$$

These three formulas, together with Theorem (1.3), say that $F^{\times} / N K^{\times}$is determined as a valued group by $k_{v}, \Gamma_{v}, v(2), v^{\mathrm{sq}}\left(d \cdot F^{\times 2}\right), v(d)+2 \Gamma_{v}$ (considered as an element of $\left.\Gamma_{v} / 2 \Gamma_{v}\right)$ and $d \cdot k_{v}^{\times 2}$, as claimed in the introduction.

This remark is proved in $\S 3$.

The reader might have noticed that Theorem (1.1) implies the well-known local squares theorem (under our hypotheses on $F$ ), namely that $1+4 \mathfrak{p}_{v} \subset F^{\times 2}$. A similar result is a consequence of (1.3). 
2. Filtered groups. Throughout this section $\Lambda$ will denote an arbitary but fixed linearly ordered set.

(2.1) Definition. Let $G$ be an (additively written) abelian group. A $\Lambda$-filter on $G$ is an indexed family $\left(G_{\lambda}\right)_{\lambda \in \Lambda}$ of subgroups of $G$ such that $G_{\lambda} \supseteq G_{\mu}$ if $\lambda \leqq \mu$. We call such a filter valued if and only if for each $a \neq 0$ in $G$ there is a largest $\lambda \in \Lambda$ with $a \in G_{\lambda}$. Finally, we say $G$ is ultracomplete with respect to the filter if and only if $G$ is complete for every (not necessarily Hausdorff) group topology admitting some family of $G_{\lambda}$ 's as a neighborhood base at zero (this is essentially the definition of Fleisher [6]).

One can check that $G$ is ultracomplete if and only if for each subset $\Omega$ of $\Lambda$ any family of cosets $\left\{a_{\lambda}+G_{\lambda} \mid \lambda \in \Omega\right\}$ with the finite intersection property (i.e. finite subfamilies have nonvoid intersection) has nonvoid intersection. Such a family of cosets is linearly ordered (since for $\lambda \leqq \mu, a_{\lambda}+G_{\lambda}$ and $a_{\mu}+G_{\mu}$ have a common element if and only if $a_{\lambda}-a_{\mu} \in G_{\lambda}$, i.e. $a_{\lambda}+G_{\lambda} \supset a_{\mu}+G_{\mu}$ ); hence we need only consider well-ordered subsets $\Omega$ of $\Lambda$.

(For conditions equivalent to ultracompleteness for valued filters, e.g. pseudoconvergence completeness, see Theorem 1 of [6].)

There is a natural bijection between the valued $\Lambda$-filters on $G$ and the $\Lambda$-valuations on $G$. Namely for each $\Lambda$-valuation $v$ we have the valued $\Lambda$-filter

$$
(\{a \in G \mid v(a) \geqq \lambda\})_{\lambda \in \Lambda},
$$

and for each valued $\Lambda$-filter $\left(G_{\lambda}\right)_{\lambda \in \Lambda}$ we obtain a $\Lambda$-valuation by mapping each $0 \neq a \in G$ to the largest $\lambda \in \Lambda$ with $a \in G_{\lambda}$. Our motivation for using filters and not simply valuations (or equivalently, valued filters) is that a factor group of a $\Lambda$ valued group possesses a canonical $\Lambda$-filter which is not necessarily valued.

(2.2) Example. Let $w: E \rightarrow \Gamma \cup\{\infty\}$ be a field valuation. $E$ is linearly compact at $w$ if and only if $E^{+}$is ultracomplete with respect to the filter induced by $w$, namely

$$
E_{\gamma}^{+}=\{a \in E \mid w(a) \geqq \gamma\} \quad(\gamma \in \Gamma) .
$$

The filter associated with the valuation $w^{\times}: E^{\times} \rightarrow \Lambda_{w} \cup\{\infty\}$ (see Example (B) of §1) has $E_{0^{*}}^{\times}=E^{\times}, E_{0}^{\times}=$units of the valuation ring of $w$, and $E_{\gamma}^{\times}=1+E_{\gamma}^{+}$for $\gamma>0$. (Since when $\gamma>0, E_{\gamma}^{+}$is an ideal of the valuation ring of $w, E_{\gamma}^{\times}$is what is classically called the "ray modulo $E_{\gamma}^{+}$. .") The reader can check that $E^{\times}$is ultracomplete for its filter if and only if $E$ is linearly compact at $w$ (use the corresponding fact for $E^{+}$and that for $a, b \in E^{\times}$with $\left.w(a)=w(b)=0, w^{\times}\left(a b^{-1}\right)=w(a-b)\right)$.

We motivate the next three propositions by suggesting their application to $F^{\times} / F^{\times 2}$. Proposition (2.3) says that $F^{\times} / F^{\times 2}$ is valued and ultracomplete if $F^{\times 2}$ is ultracomplete. This latter fact will follow from Proposition (2.4) (plus some trickery). Proposition (2.5) then applies to give the structure of $F^{\times} / F^{\times 2}$ in terms of an associated graded group.

(2.3) Proposition. Let $C$ be a subgroup of the $\Lambda$-filtered group $G$ (notation as in (2.1)). We give $C$ and $G / C$ their natural $\Lambda$-filters (namely, for each $\lambda \in \Lambda$, take 
$C_{\lambda}=C \cap G_{\lambda}$ and $\left.(G / C)_{\lambda}=\left(G_{\lambda}+C\right) / C\right)$. Then $G / C$ is valued and ultracomplete if both $G$ and $C$ are valued and ultracomplete.

Proof. Let $\Omega$ be a well-ordered subset of $\Lambda$ and let

$$
\mathscr{A}=\left\{\left(a_{\omega}+C\right)+(G / C)_{\omega} \mid \omega \in \Omega\right\}
$$

be a family with the finite intersection property; we must show $\bigcap \mathscr{A}$ is nonempty (see the remarks after Definition (2.1)). Since $G$ is ultracomplete, it suffices to construct a family

$$
\mathscr{B}=\left\{b_{\omega}+G_{\omega} \mid \omega \in \Omega\right\}
$$

having the finite intersection property and having $b_{\omega}-a_{\omega} \in C$ for all $\omega \in \Omega$ (for then $b+C \in \bigcap \mathscr{A}$ for any $b \in \bigcap \mathscr{B})$. We construct such a $\mathscr{B}$ by transfinite induction; say for some $\tau \in \Omega$ we have a family

$$
\mathscr{B}_{\tau}=\left\{b_{\omega}+G_{\omega} \mid \omega \in \Omega, \omega<\tau\right\}
$$

with the finite intersection property and with $b_{\omega}-a_{\omega} \in C$ for $\omega<\tau$. Then for any fixed $b \in \bigcap \mathscr{B}_{\tau}$ we can write for each $\omega<\tau, b-a_{\tau}=g_{\omega}+c_{\omega} \in G_{\omega}+C$. Since $C$ is ultracomplete we can find $c$ in $\bigcap\left\{c_{\omega}+C_{\omega} \mid \omega \in \Omega, \omega<\tau\right\}$. It suffices to set $b_{\tau}$ $=a_{\tau}+c$.

Next suppose $g+C \neq 0$ is in $G / C$. We show

$$
\Omega=\left\{\lambda \in \Lambda \mid g+C \in(G / C)_{\lambda}\right\}
$$

has a largest element. If for each $\omega \in \Omega$ we write $g=g_{\omega}+c_{\omega} \in G_{\omega}+C$ then since $C$ is ultracomplete we can find $c$ in $\bigcap\left\{c_{\omega}+C_{\omega} \mid \omega \in \Omega\right\}$. But clearly, $w(g-c)$ is the maximum of $\Omega$ (since $g-c \in G_{\alpha}+C$ for all $\alpha \in \Omega$ ).

Let $G$ be a $\Lambda$-filtered group with filter $\left(G_{\lambda}\right)_{\lambda \in \Lambda}$. Associated with this filter is the graded group

$$
\Delta_{\lambda}(G)=G_{\lambda} / \bigcup_{\beta>\lambda} G_{\beta} \quad(\lambda \in \Lambda)
$$

which we denote by $\Delta(G)$. A group homomorphism $f: G \rightarrow G^{\prime}$ with $f\left(G_{\lambda}\right) \subset G_{\lambda}^{\prime}$ for all $\lambda \in \Lambda$ (i.e. a "morphism of filtered groups"; here $\left(G_{\lambda}^{\prime}\right)_{\lambda \in \Lambda}$ is a given $\Lambda$-filter on $G^{\prime}$ ) induces a homomorphism of graded groups $\Delta(f): \Delta(G) \rightarrow \Delta\left(G^{\prime}\right)$.

(2.4) Proposition. Let $f: G \rightarrow G^{\prime}$ be as above. Suppose $G$ is ultracomplete and both $G$ and $G^{\prime}$ are valued (for their respective filters). Then if $\Delta(f)$ is surjective, $f$ is surjective and $G^{\prime}$ is ultracomplete.

Proof. Let $\lambda \in \Lambda, x \in G_{\lambda}^{\prime}$. By transfinite induction and the fact that for all $\alpha \in \Lambda$

$$
G_{\alpha}^{\prime}=f\left(G_{\alpha}\right)+\bigcup_{\beta>\alpha} G_{\beta}^{\prime}
$$

(since $\Delta(f)$ is surjective), we can construct $g \in G_{\lambda}$ with $f(g)=x$. (The idea is that for any "estimate" $f(g)$ for $x$, say with $f(g)-x \in G_{\alpha}^{\prime}$, we have $g^{\prime} \in G_{\alpha}$ and $\beta>\alpha$ with 
$f\left(g-g^{\prime}\right)-x \in G_{\beta}^{\prime}$; use that $G$ is ultracomplete and $G^{\prime}$ valued to show we eventually get a pre-image of $x$.) We conclude that $f\left(G_{\lambda}\right)=G_{\lambda}^{\prime}$ for each $\lambda \in \Lambda$. Hence $f$ is surjective since $G^{\prime}$ is valued. Moreover, $G^{\prime}$ may be identified with $G / \operatorname{ker} f$ as a $\Lambda$-filtered group. Hence by the previous proposition, $G^{\prime}$ is ultracomplete if $\operatorname{ker} f$ is ultracomplete.

So suppose we have a family of cosets

$$
\mathscr{A}=\left\{a_{\omega}+\operatorname{ker} f \cap G_{\omega} \mid \omega \in \Omega\right\}
$$

(each $a_{\omega} \in \operatorname{ker} f, \Omega$ a subset of $\Lambda$ ) with the finite intersection property. Since $G$ is ultracomplete we have an $a \in \bigcap\left\{a_{\omega}+G_{\omega} \mid \omega \in \Omega\right\}$. There exists $\gamma \in \Lambda$ greater than or equal to any element of $\Omega$ and such that $f(a) \in G_{\gamma}^{\prime}$ (since $G^{\prime}$ is valued and $f(a)$ $=f\left(a-a_{\omega}\right) \in G_{\omega}^{\prime}$ for all $\left.\omega \in \Omega\right)$. By the first paragraph we can find $b \in G_{\gamma}$ with $f(a)$ $=f(b)$. Clearly $b-a \in \operatorname{ker} f$ and $b-a \in \bigcap \mathscr{A}$.

We state for reference a well-known result which describes a situation in which $\Delta(G)$ completely determines $G$.

(2.5) Proposition. Let $G$ be a $\Lambda$-filtered group. Assume $G$ is valued, ultracomplete, and has prime exponent. Then $G$ is isomorphic as a valued group to the well-ordered product $\mathbf{H}_{\lambda \in \Lambda} \Delta_{\lambda}(G)$ (cf. Example (C) of $\left.\S 1\right)$.

The existence of a valuation-preserving embedding $f$ of $G$ into the well-ordered product with $\Delta(f)$ surjective is due to Conrad [5]. That this embedding is surjective follows from (2.4) above (or use Theorem 1 of [6] to apply Gravett [8, p. 312]).

We have proved only those results on $\Lambda$-filtered groups that we need for the calculations below. For many further results see [10].

3. Proofs of main theorems. Let $F$ and $v$ be as in the first section. The following conventions will allow us to discuss (1.1) and (1.3) simultaneously.

Let $K$ be either $F$ or a quadratic extension of $F$. Let $G=\{1, \sigma\}$ be the group with two elements. The unique homomorphism of $G$ onto the Galois group of the extension $K / F$ induces an operation of $G$ on $K$ (equivalently, $G$ is the Galois group if $K \neq F$, and both 1 and $\sigma$ operate as the identity on $K$ if $K=F$ ). If we define $N: K \rightarrow F$ by $N(a)=a a^{\sigma}$ (we write $a^{\sigma}$ for $\sigma(a)$ ), then $F^{\times} / N K^{\times}$is the norm factor group when $K \neq F$ and the square factor group when $K=F$.

The valuation $v$ has a unique extension, call it $w$, to $K$. Notice we have " $e f=n$ ", i.e.

$$
[K: F]=\left(\Gamma_{w}: \Gamma_{v}\right)\left[k_{w}: k_{v}\right]
$$

We denote by $N: k_{w} \rightarrow k_{v}$ the map induced by $N: K \rightarrow F$. Notice for each $a \in K$, $w(a)=w\left(a^{\sigma}\right)$ so $v(N(a))=2 w(a)$.

Recall the linearly ordered set

$$
\Lambda_{v}=\left\{0^{*}\right\} \cup\left\{\alpha \in \Gamma_{v} \mid \alpha \geqq 0\right\}
$$


of $\S 1$; we define $\Lambda_{w}$ analogously (i.e. replace $v$ above by $w$ ) and we identify $\Lambda_{v}$ with its canonical image in $\Lambda_{w}$. Finally, we give $F^{\times}$and $K^{\times}$the valuations $v^{\times}$and $w^{\times}$which are induced by $v$ and $w$, respectively (notation is as in Example (B) of $\S 1$ ). Recall that if $v(a)=0, v^{\times}(a)=v(1-a)$.

(3.1) Lemma. A. The set $\left\{w^{\times}(t) \mid t \in \operatorname{ker} N\right\}$ has a minimum, which we denote by $\lambda=\lambda_{K \mid F}$. Moreover, $0 \leqq \lambda \leqq v(2)$, and $\lambda \neq 0$ if and only if $k_{v}$ has characteristic two and $k_{v}=k_{w}$.

B. If $S: K^{+} \rightarrow F^{+}$is the trace map (i.e. $\left.a \mapsto a+\sigma(a)\right)$, then $w(S(t)) \geqq w(t)+\lambda$. Finally, there exists $a_{0} \in K^{\times}$with

$$
w\left(N\left(a_{0}\right)\right)=2 w\left(a_{0}\right)=2 \lambda, \quad N\left(a_{0}\right)=-S\left(a_{0}\right) .
$$

Proof. If $K=F$ the lemma is immediate $(\operatorname{ker} N=\{ \pm 1\}, \lambda=v(2)$, and we can take $\left.a_{0}=-2\right)$.

Suppose $K \neq F$. By Hilbert's Theorem 90, the elements of ker $N$ are exactly those of the form $t^{\sigma} t^{-1}$ for some $t \in K^{\times}$; for any $t \in K^{\times}$we have $w^{\times}\left(t^{\sigma} t^{-1}\right)=w\left(\left(t^{\sigma}-t\right) / t\right)$. The lemma now follows from MacKenzie-Whaples [14] and Geissinger [7] (set $a_{0}=t^{\sigma} t^{-1}-1$ and $\lambda=w\left(a_{0}\right)$ for any orthogenerator $t$ of $\left.K / F\right)$.

We now define a filter on $N K^{\times}$with respect to which the map $N: K^{\times} \rightarrow N K^{\times}$ is a morphism of filtered groups in the sense of Proposition (2.4) (where $K^{\times}$has the filter induced by $\left.w^{\times}\right)$. Define $\theta: \Lambda_{w} \rightarrow \Lambda_{w}$ by $\theta(\alpha)=\alpha+\min (\alpha, \lambda)\left(\right.$ recall $\alpha+0^{*}$ $=\alpha$ for $\alpha \in \Lambda_{w}$ ); note $\theta$ is strictly increasing.

(3.2) Lemma. For each $\alpha \in \Lambda_{w}$, let $L_{\alpha}=N K^{\times} \cap K_{\theta(\alpha)}^{\times}$. Then $N: K^{\times} \rightarrow N K^{\times}$is a morphism of filtered groups where $K^{\times}$has the filter induced by $w^{\times}$and $N K^{\times}$has the filter $\left(L_{\alpha}\right)_{\alpha \in \Lambda_{w}}$. Moreover, for any $b \in K^{\times}, w^{\times}(N(b))=\theta\left(w^{\times}(b)\right)$ if either $w^{\times}(b)<\lambda$ or $w^{\times}(N(b)) \leqq 2 \lambda$.

Proof. We suppose without loss of generality that $w^{\times}(b) \geqq 0$. Then $w(N(b))=0$ and using Lemma (3.1),

$$
\begin{aligned}
w^{\times}(N(b)) & =w\left(1-b^{\sigma} b\right)=w(1-b)+w\left(2-(1-b)+b\left((1-b)^{\sigma}(1-b)^{-1}-1\right)\right) \\
& \geqq w^{\times}(b)+\min \left(v(2), w^{\times}(b), \lambda\right)=\theta\left(w^{\times}(b)\right) .
\end{aligned}
$$

Notice that equality holds above when $w^{\times}(b)<\lambda$. The lemma now follows immediately.

The next lemma will be used to show the surjectivity of $\Delta(N)$ (for the above filter on $N K^{\times}$).

(3.3) LemmA. Suppose $\alpha \in \Lambda_{w}$ with $\alpha>\lambda$ and $\theta(\alpha) \in \Lambda_{v}$. Then

$$
N\left(K_{\alpha}^{\times}\right) \cdot \bigcup_{\beta>\alpha}\left(F^{\times} \cap K_{\theta(\beta)}^{\times}\right)=F^{\times} \cap K_{\theta(\alpha)}^{\times} .
$$

Proof. By definition of $\theta, \theta\left(\Lambda_{w}\right)$ contains every $\beta \in \Lambda_{w}$ with $\beta>2 \lambda$. Hence it suffices to find, for each $b \in F^{\times}$with $w^{\times}(b)=\theta(\alpha)$, some $z \in K_{\alpha}^{\times}$such that

$$
b / N(z) \in \bigcup_{\beta>\alpha} F^{\times} \cap K_{\theta(\beta)}^{\times} .
$$


Let $a_{0}$ be as in $B$ of (3.1). It suffices to take $z=1+(b-1) S\left(a_{0}\right)^{-1} a_{0}$. (Details. Using (3.1) and the definition of $\theta$,

$$
\begin{aligned}
w^{\times}(z) & =w(b-1)-w\left(S\left(a_{0}\right)\right)+w\left(a_{0}\right) \\
& =w(b-1)-\lambda=\theta(\alpha)-\lambda=\alpha .
\end{aligned}
$$

Also by the choice of $a_{0}$,

$$
\begin{aligned}
w^{\times}(N(z) / b) & =w^{\times}\left(1+b^{-1} N\left(a_{0} S\left(a_{0}\right)^{-1}(b-1)\right)\right) \\
& =2 w\left(a_{0} S\left(a_{0}\right)^{-1}(b-1)\right)=2(\theta(\alpha)-\lambda)>\theta(\alpha)
\end{aligned}
$$

which was to be proved.)

(3.4) LemMA. $N K^{\times}$is ultracomplete and valued with respect to the filter induced by the filter on $F^{\times}$(i.e. $\left(N K^{\times}\right)_{\lambda}=F_{\lambda}^{\times} \cap N K^{\times}$for each $\lambda \in \Lambda_{v}$ ).

Proof. We will use Lemma (3.2) repeatedly. First note

$$
w^{\times}\left(N K^{\times}\right) \subset \theta\left(\Lambda_{w}\right)
$$

so that $N K^{\times}$is valuated with respect to the filter $\left(L_{\alpha}\right)_{\alpha \in \Lambda_{w}}$ of (3.2) (the associated valuation assigns to each $b \in N K^{\times}$the unique $\alpha \in \Lambda_{w}$ with $w^{\times}(b)=\theta(\alpha)$ ). Also, we have for each $\alpha \leqq \lambda$,

$$
N\left(K_{\alpha}^{\times}\right) \cdot \bigcup_{\beta>\alpha} L_{\beta}=L_{\alpha}
$$

(indeed if $N(b)$ is in $L_{\alpha}$ but not in $\bigcup_{\beta>\alpha} L_{\beta}$ then $w^{\times}(b)=\alpha$ ). The formula (2) also holds if $\alpha>\lambda$, by Lemma (3.3). Hence the morphism $N: K^{\times} \rightarrow N K^{\times}$has $\Delta(N)$ surjective. We conclude from Proposition (2.4) that $N K^{\times}$is ultracomplete with respect to the " $L_{\alpha}$ "-filter.

To show $N K^{\times}$ultracomplete for the filter induced by $F^{\times}$, let

$$
\mathscr{A}=\left\{a_{\omega} \cdot\left(N K^{\times}\right)_{\omega} \mid \omega \in \Omega\right\}
$$

be a family of cosets in $N K^{\times}$with the finite intersection property; we may assume $\Omega$ is a well-ordered subset of $\Lambda_{v}$ (see the remarks after Definition (2.1)). We may assume without loss of generality that $\Omega$ has no largest element and that $\omega \neq \tau$ in $\Omega$ implies $G_{\omega} \neq G_{\tau}$. Thus from (1) we have

$$
\left(N K^{\times}\right)_{\omega}=\bigcup L_{\beta} \quad \text { (union over } \beta \in \Lambda_{\omega}, \theta(\beta) \geqq \omega \text { ) }
$$

for any $\omega \in \Omega$. Hence for each $\omega \in \Omega$ we can choose $s(\omega) \in \Lambda_{w}$ with (recall $\left.w^{\times}\left(a_{\omega} / a_{\omega+1}\right) \in \theta\left(\Lambda_{w}\right)\right)$

$$
\left(N K^{\times}\right)_{\omega} \supseteq L_{s(\omega)} \supseteq\left(N K^{\times}\right)_{\omega+1} \cup\left\{a_{\omega} / a_{\omega+1}\right\}
$$

(here $\omega+1$ denotes $\min \{\tau \in \Omega \mid \tau>\omega\}$ ). The family $\mathscr{B}=\left\{a_{\omega}+L_{s(\omega)} \mid \omega \in \Omega\right\}$ has the finite intersection property. By the above paragraph $\mathscr{B}$, and hence $\mathscr{A}$, has nonempty intersection; the lemma is proved. 
Since $N K^{\times}$and $F^{\times}$are each ultracomplete and valued, by Proposition (2.3) we have that $F^{\times} / N K^{\times}$is an ultracomplete and valued $\Lambda_{v}$-filtered group, with valuation

$$
a \cdot N K^{\times} \mapsto \max \left\{v^{\times}(a \cdot N(b)) \mid b \in K^{\times}\right\} .
$$

Since it clearly has prime exponent (namely, 2), by Proposition (2.5) we have a value-preserving isomorphism

$$
F^{\times} / N K^{\times} \rightarrow \mathbf{H}_{\gamma \in \Lambda_{v}} \Delta_{\gamma}\left(F^{\times} / N K^{\times}\right),
$$

where the graded group $\Delta\left(F^{\times} / N K^{\times}\right)$is defined as in $\S 2$.

It remains to show that the graded group $\Delta\left(F^{\times} / N K^{\times}\right)$is isomorphic to the graded group $\Delta^{\mathrm{sq}}(F)$ when $K=F$ and to the graded group $\Delta^{N}(K / F)$ when $K \neq F$. By Noether's isomorphism theorem, for each $\gamma, \Delta_{\gamma}\left(F^{\times} / N K^{\times}\right)$is isomorphic to

$$
\Delta_{\gamma}=F_{\gamma}^{\times} /\left(F_{\gamma}^{\times} \cap N K^{\times}\right) \cdot \bigcap_{\beta>\gamma} F_{\beta}^{\times} .
$$

We proceed by cases. As earlier $\mathfrak{p}_{v}$ denotes the maximal ideal of the valuation ring of $v$.

Case $\gamma=0^{*}$. Since $\Delta_{0^{*}}=F^{\times} / F_{0}^{\times} \cdot N K^{\times}$, the map

$$
a \cdot\left(F_{0}^{\times} \cdot N K^{\times}\right) \mapsto v(a)+2 \cdot \Gamma_{w}
$$

gives an isomorphism $\Delta_{0} \cong \Gamma_{v} / 2 \cdot \Gamma_{w}$.

Case $\gamma=0$. Since $\Delta_{0}=F_{0}^{\times} / N\left(K_{0}^{\times}\right)\left(1+\mathfrak{p}_{v}\right)$, the map

$$
u \cdot\left(N K_{0}^{\times}\right)\left(1+\mathfrak{p}_{v}\right) \mapsto\left(u+\mathfrak{p}_{v}\right) \cdot N k_{w}^{\times}
$$

gives an isomorphism $\Delta_{0} \cong k_{v}^{\times} / N k_{w}^{\times}$(here $N: k_{v} \rightarrow k_{v}$ is again the map induced by $N: K \rightarrow F$ ).

Case $\gamma>2 \lambda . \Delta_{\gamma}$ is trivial (apply (3.3) with $\alpha=\gamma-\lambda$ ).

We now assume $0<\gamma \leqq 2 \lambda$. By (3.1) and our hypotheses on $F$ and $v, k_{v}$ is a perfect field of characteristic two and $k_{v}=k_{w}$.

For any $a \in F^{\times}$with $\gamma=v(a)>0$, we have an isomorphism

$$
\psi_{a}: \Delta_{\gamma}\left(F^{\times}\right) \rightarrow k_{v}^{+}
$$

given by

$$
b \cdot \bigcup_{\beta>\gamma} F_{\beta}^{\times} \mapsto(b-1) / a+\mathfrak{p}_{v} \quad\left(b \in F_{\gamma}^{\times}\right) .
$$

Thus for $\gamma>0$ it suffices to compute the image of $F_{\gamma}^{\times} \cap N K^{\times}$in $k_{v}^{+}$induced by $\psi_{a}$ (since this image is the kernel of the natural surjection $\Delta_{\gamma}\left(F^{\times}\right) \rightarrow \Delta_{\gamma}$ ).

Case $0<\gamma<2 \lambda$ and $\gamma \notin 2 \Gamma_{w}$. Since for any $a \in N K^{\times}$either $w^{\times}(a)>2 \lambda$ or $w^{\times}(a) \in 2 \Gamma_{w}$ (cf. Lemma (3.2)), we have

$$
F_{\gamma}^{\times} \cap N K^{\times} \subseteq \bigcup_{\beta>\gamma} F_{\beta}^{\times} .
$$

Hence for any $b \in F$ with $w(b)=\gamma$, the map $\psi_{b}$ induces an isomorphism $\Delta_{\gamma} \cong k_{v}^{+}$ (i.e. " $\psi_{b}\left(F_{\gamma} \cap N K\right)$ " is trivial). 
Case $0<\gamma<2 \lambda$ and $\gamma \in 2 \Gamma_{w}$. We show $\Delta_{\gamma}$ is trivial. Let $a \in F^{\times}, v^{\times}(a)=\gamma$. It suffices to find $z \in N K^{\times}$with $v^{\times}(a / z)>\gamma$. Since $k_{v}$ is perfect we can find $c \in K$ and $u \in F_{0}^{\times}$with

$$
u^{2}+\mathfrak{p}_{v}=(1-a) N(c)^{-1}+\mathfrak{p}_{v}
$$

( $c$ can be any element in $K$ with $w(N(c))=\gamma$ ). It suffices to set $z=N(1+u c)$. (Details. Since $a$ and $N(1+u c)$ are in $F_{0}^{\times}$, using (3.1) gives

$$
\begin{aligned}
v^{\times}(a / N(1+u c)) & =v\left(a-1-u^{2} N(c)-u S(c)\right) \\
& \left.\geqq \min \left\{v\left(a-1-u^{2} N(c)\right), w(c)+\lambda\right\}>\gamma .\right)
\end{aligned}
$$

Case $\gamma=2 \lambda>0$. Let $b=N\left(a_{0}\right)$ where $a_{0}$ is chosen as in B of Lemma (3.1). We claim that the image of $N K^{\times} \cap F_{\gamma}^{\times}$under $\psi_{b}$ is $\left\{u^{2}-u \mid u \in k_{v}\right\}$, i.e., $\Delta_{y} \cong k_{v}^{+} \mid\left\{u^{2}-u \mid u \in k_{v}\right\}$.

First note that for any $u \in F_{0}^{\times}$we have

$$
N\left(1+u a_{0}\right)=1+\left(u^{2}-u\right) N\left(a_{0}\right)=1+\left(u^{2}-u\right) b
$$

so $\psi_{b}\left(F_{y}^{\times} \cap N K^{\times}\right)$certainly contains $\left\{u^{2}-u \mid u \in k_{v}\right\}$. On the other hand, if $N(c) \in F_{\gamma}^{\times} \cap N K^{\times}$, say with $v^{\times}(N(c))=2 \lambda$, then we can find $u \in K_{0}^{\times}$and $u^{\prime} \in F_{0}^{\times}$ with

$$
c=1+u^{\prime} a_{0} \text { and } u-u^{\prime} \in \mathfrak{p}_{w}
$$

(as $k_{v}=k_{w}$ and by B of (3.1), $w^{\times}(c)=\lambda$ ). By applying A of (3.1) and formulas (3) and (4) above, we obtain

$$
\begin{aligned}
v^{\times}\left(N(c) \cdot\left(1+\left(u^{2}-u\right) b\right)^{-1}\right) & =v^{\times}\left(N\left(\left(1+u^{\prime} a_{0}\right) /\left(1+u a_{0}\right)\right)\right) \\
& \geqq \theta\left(w\left(u^{\prime} a_{0}-u a_{0}\right)\right)>\theta\left(w\left(a_{0}\right)\right)=2 \lambda
\end{aligned}
$$

so that $\psi_{b}(N(c))=\left(u^{2}-u\right)+\mathfrak{p}_{v}$ and we are done.

If $d \in F^{\times 2}$, then $\lambda=v(2), 2 \Gamma_{w}=2 \Gamma_{v}$, and $N k_{w}^{\times}=k_{v}^{\times 2}$, so Theorems (1.1) and (1.3) are proved.

Proposition (1.2) was proved as part of (3.1). It remains to prove Remark (1.4), i.e. to compute $\lambda_{K / F}, N k_{w}^{\times}$, and $2 \Gamma_{w}$, when $K \neq F$.

So suppose $K \neq F$ and $K=F[\sqrt{ } d]$. For any $t=a+(b / \sqrt{ } d)$ in $K$ with $b \neq 0$ (so $t \notin F$ ) we have (recall $2 w(a)=v(N(a))$ for $a \in K)$

$$
2 w\left(1-t^{\sigma} t^{-1}\right)=v(4)-v\left(1-d(a / b)^{2}\right)
$$

formula (1) of (1.4) now follows from Lemma (3.1) (check separately the cases $\left.v^{\mathrm{sq}}\left(d F^{\times 2}\right)=0^{*}, \neq 0^{*}\right)$.

Now suppose $v(d) \notin 2 \Gamma_{v}$. Then $\Gamma_{w}=\Gamma_{v}+Z \cdot(v(d) / 2)$ and $k_{w}=k_{v}$ so formulas (2) and (3) of (1.4) hold in this case (note $N$ is the squaring map and $d \cdot k_{v}^{\times 2}$ is empty).

Finally suppose $v(d) \in 2 \Gamma_{v}$. Formula (3) of (1.4) holds when $k_{v}$ has characteristic two since $N k_{w}^{\times}=k_{v}^{\times 2}=k_{v}^{\times}$(recall $k_{v}$ is perfect); it holds when $k_{v}$ does not have characteristic two since then $k_{w}$ is a proper extension of $k_{v}$ (generated by any $\sqrt{ }\left(c^{2} d+\mathfrak{p}_{v}\right)$ with $\left.v\left(c^{2} d\right)=0\right)$ and $N$ is the usual norm map. 
If $\lambda=0, v^{\mathrm{sq}}\left(d \cdot F^{\times 2}\right)=v(4)$ and $\Gamma_{v}=\Gamma_{w}$ so formula (2) of (1.4) holds (consider separately the cases when $k_{v}$ does or does not have characteristic two). Suppose $\lambda \neq 0$. By our calculation of $\Delta\left(F^{\times} / F^{\times 2}\right)$, if $v^{\mathrm{sq}}\left(d \cdot F^{\times 2}\right)<v(4)$, then $v^{\mathrm{sq}}\left(d \cdot F^{\times 2}\right)$ is not in $2 \Gamma_{v}$. By formula (1) it is not $v(4)$. Hence $\Gamma_{w}=\Gamma_{v}+Z\left(v^{\mathrm{sq}}\left(d \cdot F^{\times 2}\right) / 2\right.$ ) (say $v^{\mathrm{sq}}\left(d \cdot F^{\times 2}\right)=v\left(1-c^{2} d\right)$; then since $1-c^{2} d$ is the norm of $1-c \sqrt{ } d, v^{\mathrm{sq}}\left(d \cdot F^{\times 2}\right)$ $=2 w(1-c \sqrt{ } d)$ ). Formula (2) of (1.4) follows immediately.

This completes the proofs of the results of $\S 1$.

We thank D. K. Harrison for suggestions which lead to significant simplifications of our original proofs of (1.1) and (1.3).

4. Quaternion algebras. As an application of the preceding results we study the central simple algebras over $F$ with dimension 4 ; the hypotheses on $F$ remain as in $\S 1$. By Wedderburn's theorem and [1, p. 74, Theorem 9] such algebras are exactly the generalized quaternion algebras over $F$, i.e. those $F$-algebras admitting a basis over $F$ of the form $1, x, y, x y$ where for some $c$ and $d$ in $F^{\times}$

$$
x^{2}=c \cdot 1, \quad y^{2}=d \cdot 1, \text { and } x y=-y x .
$$

For each $c, d \in F^{\times}$we let $(c, d)$ denote the unique $F$-isomorphism class of generalized quaternion algebras over $F$ admitting a basis of the above form satisfying (1).

Let $D \in(c, d)$ be such a generalized quaternion algebra. We identify $F$ with $F \cdot 1$. For $\alpha \in D$, say $\alpha=a_{1}+a_{2} x+a_{3} y+a_{4} x y\left(a_{i} \in F\right)$, we define the "conjugate" $\alpha^{\sigma}$ of $\alpha$ to be

$$
\alpha^{\sigma}=a_{1}-a_{2} x-a_{3} y-a_{4} x y .
$$

One checks that $\sigma: a \rightarrow a^{\sigma}$ is an anti-automorphism of $D$ fixing precisely $F$ elementwise, and $\sigma^{2}$ is the identity; it is easy to show that these properties uniquely determine $\sigma$ on $D$. The "involution" $\sigma$ of $D$ permits us to define the norm map $N: D \rightarrow F$ by

$$
N(t)=t \cdot t^{\sigma} \quad(t \in D)
$$

and thence the norm factor group $F^{\times} / N D^{\times}$(here $D^{\times}$is the group of invertible elements of $D$ ).

If $D$ is not a division algebra, it is the algebra of $2 \times 2$ matrices with entries from $F$ and has trivial norm factor group (e.g. see [16, p. 146]). Therefore, we henceforth assume that $D$ is a division algebra. This is equivalent to assuming that $c$ is not a norm from the field extension $F[\sqrt{ } d] / F$ (cf. [16, 57:9]). Note in particular that $c$ and $d$ are therefore not squares in $F$.

Since $F$ is linearly compact, the valuation $v$ has a unique extension to a valuation $w$ on $D$, and further (with the obvious notation)

$$
\left[k_{w}: k_{v}\right]\left(\Gamma_{w}: \Gamma_{v}\right)=4
$$

(see [19, Chapter 2]). We define $\Lambda_{w}$ and the "valuation" $w^{\times}: D^{\times} \rightarrow \Lambda_{w} \cup\{\infty\}$ by strict analogy with Example B of $\S 1$. (By a "valuation" on a noncommutative 
group $G$ (written multiplicatively) we mean a map $u: G \rightarrow \Lambda \cup\{\infty\}, \Lambda$ a linearly ordered set, satisfying $u(a)=\infty$ iff $a=1, u(a b)=u(b a) \geqq \min \{u(a), u(b)\}$, and $u(a)$ $=u\left(a^{-1}\right)$ for $a, b \in G$; such valuations correspond to valued $\Lambda$-filters on $G$ (in the sense of §2) consisting of normal subgroups of $G$.)

(4.1) Proposition And Definition. The set $\left\{w^{\times}(t) \mid t \in \operatorname{ker} N\right\}$ has a minimum, which we denote by $\lambda=\lambda_{D / F}$.

Proof. Each $t \in D, t \notin F$, satisfies a quadratic polynomial over $F$, namely $x^{2}-\left(t+t^{\sigma}\right) x+t \cdot t^{\sigma}$. As $\sigma$ fixes $F$ and $t^{\sigma} \in F[t], \sigma$ restricts to the unique nontrivial $F$-automorphism of $F[t]$. Hence $N$ restricts to the usual norm map of the extension $F[t] / F$. Now applying Hilbert's Theorem 90 to each of the subfields $F[t]$ we find

$$
\operatorname{ker} N=\left\{t^{\sigma} t^{-1} \mid t \in D^{\times}\right\}
$$

Because of (2) we can pick $x_{1}, x_{2}, x_{3}, x_{4}$ in $D^{\times}$with

$$
w\left(\sum_{i} a_{i} x_{i}\right)=\min _{i} w\left(a_{i} x_{i}\right) \quad\left(a_{i} \in F\right)
$$

(the existence of such an "orthobasis" is proved as in [14, Proposition 1]). Now one can check directly that $w^{\times}\left(t^{\sigma} t^{-1}\right)=w\left(\left(t-t^{\sigma}\right) / t\right)$ takes its minimum for $t$ equal to some $x_{i}$. The proposition is proved.

We can now define the $\Delta_{v}$-graded group $\Delta^{N}(D / F)$ by strict analogy with the definition of $\Delta^{N}(K / F)$ in $\S 1$ (i.e. replace $K$ by $D$ and $\lambda_{K / F}$ by $\lambda_{D / F}$ ). We then have

(4.2) THEOREM. There exists a value preserving isomorphism

$$
F^{\times} / N D^{\times} \cong \mathbf{H} \Delta^{N}(D / F)
$$

where the valuation on $F^{\times} / N D^{\times}$is the map to $\Lambda_{v} \cup\{\infty\}$ given by

$$
c \cdot N D^{\times} \rightarrow \max \left\{v^{\times}(a) \mid a \in c \cdot N D^{\times}\right\}
$$

and $\mathbf{H} \Delta^{N}(D / F)$ has its canonical valuation.

Proof. The proof of (4.1) closely follows that of (1.3) given in §3. Lemma (3.1), (3.2) and (3.3) all hold with $K$ replaced by $D$. Specifically, the analogue to the first part of (3.1)A is the proposition above, while the second part generalizes readily (consider separately the cases $k_{w}=k_{v}$ and $k_{w} \neq k_{v}$ ). That the first part of (3.1)B holds when $K$ is replaced by $D$ follows from the definition of $\lambda_{D / F}$ and the trace map $S$ (namely $S(t)=t+t^{\sigma}$ for $t \in D$ ). For the second part set $a_{0}=t^{\sigma} t^{-1}-1$ where $t \in D$ has $\lambda_{D / F}=w^{\times}\left(t^{\sigma} t^{-1}\right) . \theta$ may now be defined just as in $\S 3$ (replacing $\lambda_{K / F}$ by $\lambda_{D / F}$ ) and the same proofs used to establish the $D$-analogues of Lemmas (3.2) and (3.3).

Next note that Proposition (2.4) holds when the groups in question are not necessarily commutative, provided we require the filters to consist only of normal subgroups. The proof of (3.4) then yields that $F^{\times} / N D^{\times}$is an ultracomplete valued group, the valuation as described above. 
It remains to show $\Delta\left(F^{\times} / N K^{\times}\right)$is isomorphic to $\Delta^{N}(D / F)$. This calculation goes through exactly as in $\S 3$, using the analogues of (3.1), (3.2), and (3.3).

Note. $k_{w}$ is isomorphic either to $k_{v}$ or to a quadratic extension of $k_{v}$ or to a generalized quaternion division algebra over $k_{v}$. This last possibility cannot occur when $k_{v}$ has characteristic two (because we assume $k_{v}$ is then perfect). Whenever $k_{w} \neq k_{v}, N$ induces the usual norm map from $k_{w}$ to $k_{v}$.

(4.3) Remark. Suppose $k_{v}$ and $\Gamma_{v}$ have unique extensions $k$ and $\Gamma$ with $\left[k: k_{v}\right]$ $=2$ and $\left(\Gamma: \Gamma_{v}\right)=2$, and suppose that -1 is a sum of two squares in $k_{v}$ (this last condition is equivalent to requiring the norm map from $k$ to $k_{v}$ to be surjective; for equivalent conditions see [7, p. 427]). Then the norm factor group of any quadratic extension of $F$ has exactly two elements. Moreover, there is a unique (up to $F$-isomorphism) quaternion division algebra $F$ (and hence a Hilbert symbol; see $[16, \S 63]$ where this is proved for classical local fields). As remarked byKaplansky (cf. [17, p. 137]) it follows that any quadratic form of dimension greater than four is isotropic and any equivalence class of quadratic forms is determined by its dimension, discriminant, and Hasse algebra (cf. [16, §63] or [17]).

Proof. In this and the next remark we use the fact that for each $b \in N F[y]^{\times}$ $\left(=N F[\sqrt{ } d]^{\times}\right)$and $a \in F^{\times}$we have

$$
(c, d)=(b c, d)=\left(c, a^{2} d\right)=(d, c) .
$$

(E.g. to prove the first equality replace the basis $1, x, y, x y$ by the basis $1, x\left(b_{0}+b_{1} y\right), y, x\left(b_{0}+b_{1} y\right) \cdot y$ where $b=N\left(b_{0}+b_{1} y\right)$.)

If $K$ is a quadratic extension of $F$, then by Theorem (1.3),

$$
\begin{aligned}
F^{\times} / N K^{\times} & \cong \Delta^{N}(K / F)_{0^{*}} \quad \text { if } K / F \text { is unramified, } \\
& \cong \Delta^{N}(K / F)_{v(4)} \quad \text { if } K / F \text { is ramified, }
\end{aligned}
$$

which groups have order two by hypothesis. (By $K / F$ ramified we mean of course that the value group of the unique extension of $v$ to $K$ differs from $\Gamma_{v}$.)

Now choose any $c_{1}, d_{1} \in F^{\times}$with $v^{\times}\left(c_{1}\right)=v^{\mathrm{sq}}\left(c_{1} F^{\times 2}\right)=v(4)$ and $v^{\times}\left(d_{1}\right)=v^{\mathrm{sq}}\left(d_{1} F^{\times 2}\right)$ $=0^{*}$. This is possible since by hypothesis $\Delta^{\mathrm{sq}}(F)_{\gamma}$ has order two if $\gamma=v(4)$ or $\gamma=0^{*}$. We will show that for any class $(c, d)$ of quaternion division algebras we have $(c, d)=\left(c_{1}, d_{1}\right)$, proving the above remark.

If $v(d) \in 2 \Gamma_{v}$ then $F[\sqrt{ } c] \cong F[x]$ is ramified over $F$ (otherwise by the above calculation of the norm factor group of $F[\sqrt{ } c] / F, d \in N F[\sqrt{ } c]^{\times}$contradicting that $D$ is a division algebra). Thus there exists $b \in F[\sqrt{ } c]$ with $v(N(b)) \notin 2 \Gamma_{v}$. Replacing $d$ by $d N(b)$, we may assume $v(d) \notin 2 \Gamma_{v}$.

Thus $F[\sqrt{ } d]$ is ramified over $F$. Applying the above calculation of the norm factor group with $K=F[\sqrt{ } d]$, we have $v^{N}\left(c N K^{\times}\right)=v(4)$, say with $v^{\times}(c N(t))$ $=v(4)\left(t \in K^{\times}\right)$. Since $\Delta^{\mathrm{sq}}(F)_{v(4)}$ has order two, $c_{1}$ and $c N(t)$ have product a square in $F$. Thus $(c, d)=(c N(t), d)=\left(c_{1}, d\right)$.

Applying (1.4), we see $F$ and $F\left[\sqrt{ } c_{1}\right]$ have the same value group. Since $v\left(d_{1}\right)$ has odd value in $\Gamma_{v}, d_{1}$ cannot then be a norm from $F\left[\sqrt{ } c_{1}\right]$. Since then $d_{1}$ and $d$ must 
have value $0^{*}$ (in $\left.F^{\times} / N F\left[\sqrt{ } c_{1}\right]^{\times}\right)$and $\Delta^{N}\left(F\left[\sqrt{ } c_{1}\right) / F\right)_{0^{*}}$ has two elements, $d_{1}$ and $d$ have product a norm from $F\left[\sqrt{ } c_{1}\right]$. That is, $(c, d)=\left(c_{1}, d_{1}\right)$ and the remark is proved.

(4.4) Remark. With $D$ as in Theorem (4.2) we have formulas analogous to (1) and (2) of (1.4):

$$
\begin{gathered}
2 \Gamma_{w}=2 \Gamma_{v}+Z \cdot v(c)+Z \cdot v(d)+Z \cdot v^{N}\left(c N K^{\times}\right)+Z \cdot v^{\mathrm{sq}}\left(d \cdot F^{\times 2}\right), \\
v(4)=v^{N}\left(c \cdot N K^{\times}\right)+v^{\mathrm{sq}}\left(d \cdot F^{\times 2}\right)+2 \lambda_{D / F},
\end{gathered}
$$

where $K=F[\sqrt{ } d]$ and $v^{\mathrm{sq}}$ and $v^{N}$ are the valuations of Theorem (1.1) and (1.3) (and where $Z \cdot 0^{*}=0^{*}$, and as before $0^{*}+\alpha=\alpha+0^{*}=\alpha$ for all $\alpha \in \Gamma_{v} \cup\left\{0^{*}\right\}$ ).

Proof (Sketch). We use the results of $\S 1$ and Lemma (3.1)A repeatedly (and often implicitly). We may assume without loss of generality that $v^{\times}(d)=v^{\mathrm{sq}}\left(d \cdot F^{\times 2}\right)$ and $v^{\times}(c)=v^{N}\left(c \cdot N K^{\times}\right)=v^{\mathrm{sq}}\left(c \cdot F^{\times 2}\right)$ (see the first remarks in the preceding proof and use (2) of (1.4)). We identify $F[\sqrt{ } c]$ and $K=F[\sqrt{ } d]$ with $F[x]$ and $F[y]$ in $D$ and let $\Gamma_{c}$ and $\Gamma_{d}$ denote the respective value groups. Using (2) of (1.4), (I) and (II) become respectively

$$
\begin{aligned}
& 2 \Gamma_{w}=2 \Gamma_{c}+2 \Gamma_{d}, \\
& v(4)=v^{\times}(c)+v^{\times}(d)+2 \lambda_{D / F} .
\end{aligned}
$$

Note. By the calculations of $\Delta\left(F^{\times} / N K^{\times}\right)$and $2 \Gamma_{c}, 2 \Gamma_{c} \subseteq 2 \Gamma_{d}$ if and only if $v^{\times}(c)=2 \lambda_{K / F}$.

To prove $\left(\mathrm{I}^{\prime}\right)$ we consider two cases.

Case 1. $\Gamma_{d} \neq \Gamma_{v}$. It suffices to consider the possibility that $\Gamma_{c} \subseteq \Gamma_{d}$, whence $v^{\times}(c)=2 \lambda_{K / F}$. Then $v^{\times}(c)+v^{\times}(d)=v(4)$ by (1) of (1.4). If $v^{\times}(d)=0^{*}$, then $v^{\times}(c)$ $=v(4)$; otherwise $v^{\times}(d) \geqq 0$ and $v^{\times}(c+d-c d)=v(4)$. Since neither $c$ nor $c+d-c d$ (=the square of $x+y+x y$ ) are squares in $F$, either $F[x]$ or $F[x+y+x y]$ is then unramified over $F$ (use (1) of (1.4) to apply (3.1)A). Hence $k_{v} \neq k_{w}$. Then by (2) above one must have $\Gamma_{d}=\Gamma_{w}$, proving $\left(\mathrm{I}^{\prime}\right)$.

Case 2. $\Gamma_{d}=\Gamma_{v}$. Then $K=F[y]$ is unramified over $F$, so $k_{w} \neq k_{v}$ and $\left(\Gamma_{w}: \Gamma_{v}\right) \leqq 2$. Because of (2) we need only consider the case $\left[k_{w}: k_{v}\right]=2$. It suffices to show $v^{\times}(c)$ $\neq 0$, for then $\Gamma_{c}=\Gamma_{w}$ (apply the note above using that $2 \lambda_{K / F}=0$ ). If $k_{v}$ has characteristic two, then $\Delta\left(F^{\times} / N K^{\times}\right)_{0}$ is trivial, so $v^{\times}(c) \neq 0$. So suppose $k_{v}$ does not have characteristic two. Just suppose $v^{\times}(c)=0$; then $w(x)=0$ and for some $a, b \in F$, $w(a+b y-x)>0$ (since $K=F[y]$ is unramified over $F$ and $\left[k_{w}: k_{v}\right]=2$, the residue field of $K$ “is" $\left.k_{w}\right)$. Then $v^{\times}\left(c \cdot N(a+b y)^{-1}\right)>0=v(4)$ so $c \in N K^{\times}$. This contradiction proves $\left(\mathrm{I}^{\prime}\right)$.

To prove (II') we also have two cases.

Case 1. $v(4) \leqq v^{\times}(c)+v^{\times}(d)$. As argued earlier $c, d$, and $c+d-c d$ are nonsquares in $F$ and hence each has $v^{\times}$-value at most $v(4)$. When $v^{\times}(c)$ and $v^{\times}(d)$ are both unequal to $0^{*}$ we have $v^{\times}(c)+v^{\times}(d)=v^{\times}(c+d-c d)$, and hence for $b$ equal to one of $c, d$, and $c+d-c d$, we have $v^{\times}(b)=v(4)$. But then $\lambda_{F[\sqrt{ } b] / F}=0$ so necessarily $\lambda_{D / F}=0$. (II') now follows. 
Case 2. $v(4)>v^{\times}(c)+v^{\times}(d)$. Since $v^{\times}(d)<v(4), \Gamma_{d} \neq \Gamma_{v}$ (use (3.1)A). $2 \Gamma_{d}$ is generated over $2 \Gamma_{v}$ by $2 w(u)$, where $u=y$ if $v(d) \notin 2 \Gamma_{v}$ and $u=1-y$ if $v^{\times}(d) \notin 2 \Gamma_{v}$ (apply (2) of (1.4)). Further, $\Gamma_{c} \nsubseteq \Gamma_{d}$ since $v^{\times}(c)<v(4)-v^{\times}(d)=2 \lambda_{K / F}$ (applying a remark above). Hence $2 \Gamma_{c}+2 \Gamma_{d}$ is generated over $2 \Gamma_{d}$ by $2 w(t)$, where $t=x$ if $v(c) \notin 2 \Gamma_{d}$, and $t=1-x$ if $v^{\times}(c) \notin 2 \Gamma_{d}$. We then have that $1, t, u, t u$ is an orthobasis for the extension $D / F$. One now checks (II') directly, using that $2 \lambda_{D / F}$ is the minimum of $2 \cdot w\left(z^{\sigma} z^{-1}-1\right)=v\left(N\left(z^{\sigma} z^{-1}-1\right)\right)$ as $z$ ranges over an orthobasis.

Remark (4.4) is proved.

5. Not necessarily linearly compact fields. Throughout this section $F$ is a field not of characteristic two and $v$ is a valuation on $F$ such that $k_{v}$ is perfect if it has characteristic two.

Let $K=F[\sqrt{ } d]$ be a quadratic extension of $F$ and let $w$ be an extension of $v$ to $K$. Then $F^{\times} / F^{\times 2}$ and $F^{\times} / N K^{\times}$each have a $\Lambda_{v}$-filter canonically induced (cf. (2.3)) by the $\Lambda_{v}$-filter on $F^{\times}$(cf. Example (2.2)). Since we are not assuming $F$ linearly compact, neither $F^{\times} / F^{\times 2}$ nor $F^{\times} / N K^{\times}$is in general either valued or ultracomplete as a filtered group.

The key observation of this section is

(5.1) REMARK. There is an isomorphism $\Delta\left(F^{\times} / F^{\times 2}\right) \cong \Delta^{\mathrm{sq}}(F)$ (where $\Delta^{\mathrm{sq}}(F)$ is as in $\S 1$ and the $\Lambda_{v}$-graded group $\Delta\left(F^{\times} / F^{\times 2}\right)$ induced by the $\Lambda_{v}$-filter on $F^{\times} / F^{\times 2}$ is defined as in $\S 2$ ). Further, when the extension $K / F$ is not immediate (i.e. when we do not have both $k_{v}=k_{w}$ and $\left.\Gamma_{v}=\Gamma_{w}\right)$, then $\lambda_{K / F}$ exists and there is an isomorphism $\Delta\left(F^{\times} / N K^{\times}\right) \cong \Delta^{N}(K / F)$ (where $\lambda_{K / F}$ and $\Delta^{N}(K / F)$ are defined as in $\S 1$ ).

The assertions above are proved exactly as in the case that $F$ is linearly compact (cf. §3).

We will apply the above calculation of $\Delta\left(F^{\times} / F^{\times 2}\right)$ to deduce the ramification theory of the extensions of $v$ to $K$ from the behavior of $d \cdot F^{\times 2}$ in the $\Lambda_{v}$-filter on $F^{\times} / F^{\times 2}$. We will also note some hypotheses under which $F^{\times} / F^{\times 2}$ and $F^{\times} / N K^{\times}$ are valued and ultracomplete (and hence determined by their graded groups), and indicate analogous results for generalized quaternion division algebras.

Let $g$ be the number of extensions of $v$ to $K$. We remind the reader that

$$
\left(\Gamma_{w}: \Gamma_{v}\right) \cdot\left[k_{w}: k_{v}\right] \cdot g \leqq 2=[K: F] .
$$

(5.2) Remark. (A) $g=1$ if and only if $v^{\times}\left(d \cdot F^{\times 2}\right)=\left\{v^{\times}(c) \mid c \in d \cdot F^{\times 2}\right\}$ is bounded above by $v(4)$ (that is, $d F^{\times 2} \notin \Delta\left(F^{\times} / F^{\times 2}\right)_{\gamma}$ for all $\gamma>v(4)$ ).

(B) $\left[k_{w}: k_{v}\right]=2$ if and only if $v(4)$ is the maximum of $v^{\times}\left(d \cdot F^{\times 2}\right)$.

(C) $\left(\Gamma_{w}: \Gamma_{v}\right)=2$ if and only if $v^{\times}\left(d \cdot F^{\times 2}\right)$ has a maximum which is strictly less than $v(4)$.

The proof of (A) is an elementary deduction from the fact that every extension of $v$ to $K$ is of the form $w \circ \sigma, \sigma$ in the Galois group of $K$ over $F$. When $K / F$ is not immediate, $v^{\times}\left(d \cdot F^{\times 2}\right)$ has a maximum and

$$
2 \lambda_{K / F}=v(4)-\max v^{\times}\left(d \cdot F^{\times 2}\right)
$$


(argue as in the proof of (1) of (1.4)). (B) and (C) now follow from the isomorphism $\Delta\left(F^{\times} / F^{\times 2}\right) \cong \Delta^{\mathrm{sq}}(F)$. (This argument was made in the proof of (1.4); the key fact is that if $\gamma$ is the maximum of $v^{\times}\left(d \cdot F^{\times 2}\right)$, then $\Delta^{\mathrm{sq}}(F)_{\gamma}$ is nontrivial.)

Note that (A), (B), and (C) together imply that $K / F$ is not immediate if and only if $v^{\times}\left(d \cdot F^{\times 2}\right)$ has a maximum. When this is true, formulas (1), (2) and (3) of (1.4) hold (replacing $v^{\mathrm{sq}}\left(d \cdot F^{\times 2}\right)$ of course by $\max v^{\times}\left(d \cdot F^{\times 2}\right)$ ).

Let $I$ denote the isolated (i.e. convex) subgroup of $\Gamma_{v}$ generated by $v(2)$.

(5.3) TheOREM. Suppose $F$ is Henselian and I is either infinite cyclic or trivial. Then $F^{\times} / F^{\times 2}$ is valued (in its $\Lambda_{v}$-filter) and there is a value-preserving isomorphism

$$
F^{\times} / F^{\times 2} \cong \mathbf{H} \Delta^{\mathrm{sq}}(F)
$$

Proof. It suffices to show $F^{\times} / F^{\times 2}$ valued and ultracomplete (by (2.4) and (5.1)). Since $\left\{\gamma \in \Gamma_{v} \mid 0<\gamma<v(4)\right\}$ is finite, this follows from the local squares theorem (i.e. that $1+4 \mathfrak{p}_{v} \subseteq F^{\times 2}$; to prove this note that for $a \in \mathfrak{p}_{v}, x^{2}-(1+4 a)$ has a zero in $F$ since by Hensel's lemma $(1 / 4)\left((2 x+1)^{2}-(1+4 a)\right)=x^{2}+x+a$ has a zero in $\left.F\right)$.

(5.4) THEOREM. Let $F$ be as in (5.3). There is a value preserving isomorphism

$$
F^{\times} / N K^{\times} \cong \mathbf{H} \Delta^{N}(K / F)
$$

(we are implicitly asserting that $\lambda_{K / F}$ exists and $F^{\times} / N K^{\times}$is valued).

Proof. Since $F^{\times 2} \subseteq N K^{\times}, F^{\times} / N K^{\times}$is valued (argue as in the proof of (5.3)). Hence by (5.1) and (5.2) the extension $K / F$ is not immediate. The theorem may now be proved by mimicing the proof of (5.3).

(5.5) Remark. Suppose $F$ Henselian. If $v^{\times}(c)>v(4)-v^{\times}(d)$, then $c \in N K^{\times}$. For, one computes that either $c$ or $c+d-c d$ is in $1+4 \mathfrak{p}_{v} \subseteq F^{\times 2}$, whence $c \in N K^{\times}$ (note that $c+d-c d=b^{2}$ implies $c=\left(b^{2}-d\right) /(1-d)$ ).

Now suppose $K / F$ is unramified (i.e. $k_{w} \neq k_{v}$ ). By (5.1) and (5.2), $\lambda_{K / F}=0$. The above paragraph says $1+\mathfrak{p}_{v} \subseteq N K^{\times}$. (This was stated for the case $F$ linearly compact in [7, Theorem 3].) Hence $F^{\times} / N K^{\times}$is valued and isomorphic to $\mathbf{H} \Delta^{N}(K / F)$.

(5.6) Remark. Now suppose $F$ has no immediate quadratic extensions (e.g. suppose $F$ is algebraically complete in the sense of Ersov [22]). By Remark (5.2), $F^{\times} / F^{\times 2}$ is valued. Using (5.1), we conclude that if $F$ is countable, there is a value preserving isomorphism from $F^{\times} / F^{\times 2}$ onto

$$
\sum \Delta^{\mathrm{sq}}(F)=\sum_{\lambda \in \Lambda \nu} \Delta^{\mathrm{sq}}(F)_{\lambda}
$$

where the valuation on the direct sum is that induced by the valuation on $\mathbf{H} \Delta^{\mathrm{sq}}(F)$. (For the straightforward proof that a countable valued group $G$ of prime exponent is isomorphic to $\sum \Delta(G)$, see [21].) The reader may have noticed that the hypotheses of (5.3) guarantee that $F$ has no proper quadratic immediate extensions and that $\sum \Delta^{\mathrm{sq}}(F)=\mathbf{H} \Delta^{\mathrm{sq}}(F)$.

(5.7) Remark. Let $D \in(c, d)$ be a generalized quaternion division algebra over $F$. If $K=F[\sqrt{ } d]$ is not an immediate extension of $F$, one can show that $v$ has a 
unique extension to a valuation of $D$ if and only if $v^{\times}\left(c \cdot N K^{\times}\right)=\left\{v^{\times}(t) \mid t \in c \cdot N K^{\times}\right\}$ is bounded above by $2 \lambda_{K / F}$. Supposing this condition holds, let $w$ be the unique extension of $v$ to $D$; if further

$$
\left(\Gamma_{w}: \Gamma_{v}\right) \cdot\left[k_{w}: k_{v}\right]=4
$$

holds, then $\lambda_{D / F}$ exists and there is an isomorphism $\Delta\left(F^{\times} / N D^{\times}\right) \cong \Delta^{N}(D / F)$ (definitions as in $\$ 4$ ).

We conclude by noting that if $F$ satisfies the conditions of (5.3), then $v$ has a unique extension to $D$, (1) above holds and there is an isomorphism of valued groups (cf. Theorem (4.1))

$$
F^{\times} / N D^{\times} \cong \mathbf{H} \Delta^{N}(D / F)
$$

Moreover, formulas (I) and (II) of (4.4) hold (recall $F^{\times} / F^{\times 2}$ and $F^{\times} / N K^{\times}$are valued groups by (5.3) and (5.4)).

6. Appendix. Cyclic extensions of prime degree. Let $p$ be a prime number. Throughout this section $F$ is a field not of characteristic $p$, and $v$ is a valuation on $F$ such that $k_{v}$ is perfect if it has characteristic $p$. We sketch here how some of our results above generalize to this situation. Proofs are omitted; they generally follow those for the case $p=2$, with fuller use of [14] and [7].

Let $K$ be a cyclic extension of $F$ of degree $p$ and let $w$ be an extension of $v$ to $K$. Then $F^{\times} / F^{\times p}$ and $F^{\times} / N K^{\times}$each have a $\Lambda_{v}$-filter induced by the $\Lambda_{v}$-filter on $F^{\times}$ (just as in $\S 5$ ).

When the extension $K / F$ is not immediate, the set $\left\{w^{\times}(a) \mid a \in \operatorname{ker} N\right\}$ has a minimum, which we denote by $\lambda_{K / F}$ (just as in (3.1)). Define the $\Lambda_{v}$-graded group $\Delta^{p}(F)$ and, when $K / F$ is not immediate, the $\Lambda_{v}$-graded group $\Delta^{N}(K / F)$ by strict analogy with the $p=2$ case in $\S 1$. Specifically, write $\Delta^{p}(F), p \Gamma_{v}, k_{v}^{\times p}, p v(p) /(p-1)$, and $u^{p}-u$ in place of $\Delta^{\mathrm{sq}}(F), 2 \Gamma_{v}, k_{v}^{\times 2}, v(4)$, and $u^{2}-u$, respectively.

Note. If $F$ has a primitive $p$ th root of unity $\xi_{p}$, then $v\left(\xi_{p}-1\right)=v(p) /(p-1)$. Thus the strange substitution of $p v(p) /(p-1)$ for $v(4)$ is really the natural substitution of $p v^{\times}\left(\xi_{p}\right)$ for $2 v^{\times}\left(\xi_{2}\right)$.

(6.1) TheOREM. Suppose $F$ is linearly compact. Then $F^{\times} / F^{\times p}$ is valued in its $\Lambda_{v}$-filter and is isomorphic as a valued group to $\mathbf{H} \Delta^{p}(F)$.

(6.2) THEOREM. Suppose $F$ is linearly compact (so $K / F$ is not immediate). Then $F^{\times} / N K^{\times}$is valued in its $\Lambda_{v}$-filter and is isomorphic as a valued group to $\mathbf{H} \Delta^{N}(K / F)$.

(6.3) Remark. The graded group $\Delta\left(F^{\times} / F^{\times p}\right)$ associated with the $\Lambda_{v}$-filter on $F^{\times} / F^{\times p}$ (cf. (2.4)) is isomorphic to $\Delta^{p}(F)$. Similarly, when $K / F$ is not immediate, $\Delta\left(F^{\times} / N K^{\times}\right)$is isomorphic to $\Delta^{N}(K / F)$.

(6.4) Remark. Suppose $F$ contains a primitive $p$ th root of unity $\xi_{p}$, so that $K=F\left[d^{1 / p}\right]$ for some $d \in F$. If $v^{\times}\left(d \cdot F^{\times p}\right)$ has a maximum, then $K / F$ is not immediate and

$$
p \lambda_{K / F}=p v^{\times}\left(\xi_{p}\right)-\max v^{\times}\left(d \cdot F^{\times p}\right) .
$$


(6.5) Remark. Suppose $F$ satisfies the $p$ th power theorem, i.e. suppose that if $\gamma>p v(p) /(p-1)$ then $F_{\gamma}{ }^{\times} \subset F^{\times p}$. (When $F$ is Henselian, this is an immediate consequence of Krasner's lemma [18, p. 190].) Further suppose the isolated subgroup of $\Gamma_{v}$ generated by $v(p)$ is trivial or infinite cyclic. Then $K / F$ is not immediate and we have isomorphisms of valued groups

$$
F^{\times} / F^{\times p} \cong \mathbf{H} \Delta^{p}(F), \quad F^{\times} / N K^{\times} \cong \mathbf{H} \Delta^{N}(K / F) .
$$

Added in proof. The second author has applied the techniques and results of this paper to prove that if a field $F$ satisfies the hypotheses of $\S 1$ and possesses a unique unramified quadratic extension, then a quadratic extension of $F$ is determined by its group of norms (see Hoyt D. Warner, Determination of quadratic extensions of linearly compact fields by norm groups, submitted to the Proc. Amer. Math. Soc.). This result is well known for the classical local (and global) fields of characteristic not two.

\section{BIBLIOGRAPHY}

1. A. A. Albert, Structure of algebras, rev. ed., Amer. Math. Soc. Colloq. Publ., vol. 24, Amer. Math. Soc., Providence, R. I., 1961. MR 23 \#A912.

2. N. Bourbaki, Algèbre commutative. Chap. 6: Valuations, Fasc. XXX, Actualités Sci. Indust., no. 1308, Hermann, Paris, 1964. MR 33 \#2660.

3. Ron Brown, Extended prime spots (in preparation).

4. Ron Brown, D. K. Harrison and H. D. Warner, Ultracompletions at finite and infinite primes (in preparation).

5. P. Conrad, Embedding theorems for abelian groups with valuations, Amer. J. Math. 75 (1953), 1-29. MR 14, 842.

6. I. Fleischer, Completeness in valued spaces and algebras, Quart. J. Math. Oxford Ser. (2) 15 (1964), 345-348. MR 31 \#2241.

7. L. Geissinger, A reciprocity law for maximal fields, Trans. Amer. Math. Soc. 125 (1966), 422-431. MR 34 \#4244.

8. K. A. H. Gravet:. Valued linear spaces, Quart. J. Math. Oxford Ser. (2) 6 (1955), 309-315. MR 19, 385.

9. D. K. Harrison, Finite and infinite primes for rings and fields, Mem. Amer. Math. Soc. No. 68 (1966). MR 34 \#7550.

10. - Seminar at the University of Oregon, 1967/68 (mimeographed notes).

11. I. Kaplansky, Maximal fields with valuations, Duke Math. J. 9 (1942), 303-321. MR 3, 264.

12. W. Krull, Allgemeine Bewertungstheorie, J. Reine Angew. Math. 167 (1932), 160-196.

13. S. Lefschetz, Algebraic topology, Amer. Math. Soc. Colloq. Publ., vol. 27, Amer. Math. Soc., Providence, R. I., 1942. MR 4, 84.

14. R. E. MacKenzie and G. Whaples, Artin-Schreier equations in characteristic zero, Amer. J. Math. 78 (1956), 473-485. MR 19, 834.

15. E. Matlis, Decomposable modules, Trans. Amer. Math. Soc. 125 (1966), 147-179. MR 34 \#1349.

16. O. T. O'Meara, Introduction to quadratic forms, Die Grundlehren der math. Wissenschaften, Band 117, Academic Press, New York; Springer-Verlag, Berlin, 1963. MR 27 \#2485.

17. A. Pfister, Quadratic forms, Notes by A. D. McGettrick, Cambridge University, Cambridge, 1967 (Xeroxed). 
18. P. Ribenboim, Théorie des valuations, 2ième éd., Séminaire de Mathématiques Supérieures, no. 9, Presses Université de Montréal, Montréal, Que., 1968. MR 40 \#2670.

19. O. F. G. Schilling, The theory of valuations, Math. Surveys, no. 4, Amer. Math. Soc., Providence, R. I., 1950. MR 13, 315.

20. E. Witt, Theorie der quadratischen formen in beliebigen korpern, J. Reine Angew. Math. 176 (1937), 31-44.

21. Ron Brown, Valued vector spaces of countable dimension, Publ. Math. Debrecen (to appear).

22. Ju. L. Eršov, On the elementary theory of maximal normed fields, Dokl. Akad. Nauk SSSR 165 (1965), 21-23 = Soviet Math. Dokl. 6 (1965), 1390-1393. MR 32 \#7554.

University of Oregon, Eugene, Oregon 97403

Simon Fraser University, Burnaby, British Columbia, Canada

UNIVERSITY OF IlliNOIS, URBANA, ILLINOIS 61801

Current address (Brown): Department of Mathematics, University of Hawaii, Honolulu, Hawaii 96822 